\title{
THE LIOUVILLE THEOREM FOR A QUASI-LINEAR ELLIPTIC PARTIAL DIFFERENTIAL EQUATION $\left(^{(}\right)$
}

BY

\author{
ELWOOD BOHN AND LLOYD K. JACKSON
}

The classical Liouville Theorem of analytic function theory can be stated in either of two equivalent forms:

The Liouville Theorem [1, p. 98]. If $f(w)$ is analytic and bounded throughout the finite $w$-plane, then $f(w)$ is constant.

If $z(x, y)$ is a real valued function of the real variables $x$ and $y$ which is a solution of $z_{x x}+z_{y y}=0$ and is bounded either above or below throughout the finite plane, then $z(x, y)$ is a constant.

Here we are concerned with the question of whether or not the second formulation of the above theorem is valid for solutions of more general elliptic partial differential equations. In what follows the usual notation will be adopted: $p=z_{x}, q=z_{y}, r=z_{x x}, s=z_{x y}$ and $t=z_{y y}$.

The equation

$$
A(x, y, z, p, q) r+2 B(x, y, z, p, q) s+C(x, y, z, p, q) t=0
$$

is said to be quasi-linear. It is said to be elliptic with respect to a given solution $z(x, y)$ in a domain $D$ of the $x y$-plane in case $A>0$ and $B^{2}-A C<0$ for all $(x, y) \in D$ when $z, p, q$ are replaced in $A, B$, and $C$ by $z(x, y)$ and its respective first partial derivatives.

Bernstein [2] showed that if $z(x, y)$ is a bounded solution of (1) throughout the plane and if (1) is elliptic with respect to $z(x, y)$ throughout the plane, then $z(x, y)$ must be constant.

This is not quite the Liouville Theorem since the solution is assumed to be bounded, however, it is the best that can be obtained without stronger assumptions being placed on $A, B$, and $C$.This is illustrated by the elliptic equation $\left(2+4 y^{2}\right) r+4 y s+t=0$ which has $e^{x-y^{2}}$ as an entire solution which is bounded below but which is not constant.

The equation (1) is said to be uniformly elliptic in a region $D$ of the $x y$ plane in case there is a constant $\lambda>0$ such that

$$
\lambda^{-1}\left(\xi^{2}+\eta^{2}\right) \leqq A \xi^{2}+2 B \xi \eta+C \eta^{2} \leqq \lambda\left(\xi^{2}+\eta^{2}\right)
$$

for all $(\xi, \eta)$, all $(x, y) \in D$, and all $(z, p, q)$. Serrin [3] proved that, if (1) is

Received by the editors September 25, 1961.

(1) This research was partially supported by the United States Air Force through the Air Force Office of Scientific Research of the Air Research and Development Command, under contract No. AF 49(638)-506. Reproduction in whole or in part is permitted for any purpose of the United States Government. 
uniformly elliptic, its solutions satisfy a Harnack inequality. He used this result to establish the Liouville Theorem for (1) in the case of uniform ellipticity. Bers and Nirenberg [4] proved in a different way that, if equation (2) below is uniformly elliptic and $F \equiv 0$, its solutions satisfy a Harnack inequality.

Consider now the equation

$$
L[z] \equiv A(x, y, p, q) r+2 B(x, y, p, q) s+C(x, y, p, q) \iota=F(x, y, z, p, q)
$$

concerning which the following assumptions are made:

(i) $A, B$, and $C$ are continuous and have continuous first partial derivatives with respect to $p$ and $q$ for all $(x, y, p, q)$.

(ii) $A>0$ and $B^{2}-A C<0$ for all $(x, y, p, q)$. There are continuous functions $a(p, q)$ and $c(p, q)$ and a constant $d>0$ such that $A C-B^{2}>d, A(x, y, p, q)$ $\leqq a(p, q)$ and $C(x, y, p, q) \leqq c(p, q)$ for all $(x, y)$ and all $(p, q)$ with $p^{2}+q^{2} \leqq 1$.

(iii) $F(x, y, z, p, q)$ is continuous and has continuous first partial derivatives with respect to $z, p$, and $q$ with $F_{z} \geqq 0$ for all $(x, y, z, p, q)$. Given any $N>0$ there exists an $H_{N}>0$ such that

$$
|F(x, y, z, p, q)| \leqq H_{N}\left(p^{2}+q^{2}\right)
$$

for $|z| \leqq N, p^{2}+q^{2} \leqq 1$, and all $(x, y)$.

THEOREM. If equation (2) satisfies conditions (i), (ii) and (iii), then a function $z(x, y)$ which is of class $C^{(2)}$ and a solution of (2) throughout the finite plane and which is bounded on one side must be a constant.

Proof. The proof employs a modification of the methods used by Serrin [3] and is based on the following principle:

Maximum principle [5]. Let $D$ be any plane domain and consider the function $F(x, y, z, p, q, r, s, t)$ with the following assumptions:

(i) $F$ is continuous in all 8 variables in the region $T$ defined by $T \equiv\{(x, y, z, p, q, r, s, t):(x, y) \in D$ and $-\infty<z, p, q, r, s, t<\infty\}$ and

(ii) $F_{z}, F_{p}, F_{q}, F_{r}, F_{s}$, and $F_{t}$ are continuous on $T, F_{s}^{2}-4 F_{r} F_{t}<0, F_{r}>0$, and $F_{z} \leqq 0$ on $T$. Let $z_{1}(x, y)$ and $z_{2}(x, y)$ be continuous in a bounded and closed subdomain $\Omega \subset D$ and of class $C^{(2)}$ in the interior of $\Omega$. Furthermore, suppose $z_{1}(x, y) \leqq z_{2}(x, y)$ on the boundary of $\Omega$ and suppose that in the interior of $\Omega$

$$
\begin{aligned}
& F\left(x, y, z_{1}, z_{1 x}, z_{1 y}, z_{1 x x}, z_{1 x y}, z_{1 y y}\right) \geqq 0 \\
& F\left(x, y, z_{2}, z_{2 x}, z_{2 y}, z_{2 x x}, z_{2 x y}, z_{2 y y}\right) \leqq 0
\end{aligned}
$$

Then, either $z_{1}(x, y)<z_{2}(x, y)$ in the interior of $\Omega$ or $z_{1}(x, y) \equiv z_{2}(x, y)$, on $\Omega$.

The conditions placed on equation (2) are such that $L[z]-F(x, y, z, p, q)$ satisfies the hypothesis placed on $F(x, y, z, p, q, r, s, t)$ in the Maximum Principle. 
It suffices to consider the case in which $z(x, y)$ is a solution of (2) which is bounded below in the finite plane since the bounded above case can be reduced to this one by replacing $z(x, y)$ by $-z(x, y)$.

Assume that there is a nonconstant solution $z(x, y)$ of (2) which is bounded below in the finite plane and let $z_{0}=\operatorname{glb} z(x, y)$. Then $w(x, y)$ $\equiv z(x, y)-z_{0}$ is a solution of

$$
L[w]=F\left(x, y, w+z_{0}, w_{x}, w_{y}\right)
$$

throughout the finite plane and glb $w(x, y)=0$. Furthermore, it is clear that equation (3) also satisfies conditions (i), (ii), and (iii) placed on equation (2). It follows from the third condition that $F(x, y, z, 0,0) \equiv 0$ so that constants are solutions of (2). Since $z(x, y)$ is assumed to be nonconstant we can apply the Maximum Principle to conclude that $z(x, y)>z_{0}$ or $w(x, y)>0$ throughout the finite plane.

Let $K$ be the closed circular disk in the plane with center at $(0,0)$ and radius $R$. Let $E$ denote the component of the set

$$
[(x, y) \in K: w(x, y)>(1 / 2) w(0,0)]
$$

which contains $(0,0)$. We can again apply the Maximum Principle to conclude that $E$ must contain an arc of the circumference of $K$. Hence, there is a Jordan arc $\Gamma$ contained in $E$ with one end at $(0,0)$ and the other end at a point $\left(x_{0}, y_{0}\right)$ on the circumference of $K$ which is such that with the exception of $\left(x_{0}, y_{0}\right) \Gamma$ is contained in the interior of $E$. Let $K_{1}$ and $K_{2}$ be the two closed disks each of which has radius $2 R$ and each of which has the points $(0,0)$ and $\left(x_{0}, y_{0}\right)$ on its circumference.

Each point $(x, y) \in K_{1} \cap K_{2}$ satisfies at least one of the following conditions:

(a) $(x, y) \in \Gamma \cup$ bdry $\left(K_{1} \cap K_{2}\right)$,

(b) $(x, y)$ is in a subdomain of $K_{1}$ the boundary of which consists of arcs of $\Gamma$ and arcs of the circumference of $K_{1}$,

(c) $(x, y)$ is in a subdomain of $K_{2}$ the boundary of which consists of arcs of $\Gamma$ and arcs of the circumference of $K_{2}$.

Let $K_{3}$ be the closed disk with center at $\left(x_{0} / 4, y_{0} / 4\right)$ and radius $3 R / 4$ and let $\left(x_{1}, y_{1}\right)$ and $\left(x_{2}, y_{2}\right)$ be the respective centers of $K_{1}$ and $K_{2}$. It is clear that there exists a fixed $\epsilon, 0<\epsilon<1$, such that

$$
\begin{gathered}
{\left[(x, y):\left(x-x_{1}\right)^{2}+\left(y-y_{1}\right)^{2} \leqq \epsilon^{2}(2 R)^{2}\right] \subset \operatorname{comp} K,} \\
{\left[(x, y):\left(x-x_{2}\right)^{2}+\left(y-y_{2}\right)^{2} \leqq \epsilon^{2}(2 R)^{2}\right] \subset \operatorname{comp} K,} \\
{\left[(x, y):\left(x-x_{0} / 4\right)^{2}+\left(y-y_{0} / 4\right)^{2} \leqq \epsilon^{2}(3 R / 4)^{2}\right] \subset \operatorname{int}\left(K_{1} \cap K_{2}\right) .}
\end{gathered}
$$

For example, $\epsilon=3 / 128$ would do.

With a fixed $\epsilon$ satisfying the above condition, consider the function 


$$
v(x, y ; \xi, \eta, r) \equiv \frac{N\left(e^{-\alpha \sigma^{2}}-e^{-\alpha r^{2}}\right)}{1-e^{-\alpha r^{2}}}
$$

defined on the annulus

$$
S(\xi, \eta, r) \equiv\left[(x, y): \epsilon^{2} r^{2} \leqq \sigma^{2} \equiv(x-\xi)^{2}+(y-\eta)^{2} \leqq r^{2}\right]
$$

where $N=w(0,0) / 2$ and $\alpha>0$. In this annulus

$$
v_{x}^{2}+v_{y}^{2}=\frac{4 \alpha^{2} N^{2} \sigma^{2} e^{-2 \alpha \sigma^{2}}}{\left(1-e^{-\alpha r^{2}}\right)^{2}} \leqq \frac{4 N^{2}}{\sigma^{2}} \leqq \frac{4 N^{2}}{\epsilon^{2} r^{2}} \leqq 1
$$

provided $r \geqq 2 N / \epsilon$. Furthermore $v \leqq N$ on $S(\xi, \eta, r), v=0$ when $\sigma=r$, and $v>0$ when $\sigma<r$.

Let $r$ and $\beta$ be any fixed real numbers with $r \geqq 2 N / \epsilon$ and $0<\beta \leqq 1$. Then, if $A, B$, and $C$ are evaluated at $\left(x, y, \beta v_{x}, \beta v_{y}\right)$, the following succession of inequalities are valid in $S(\xi, \eta, r)$.

$$
\begin{aligned}
& \mathrm{L}[\beta v]-F\left(x, y, \beta v+z_{0}, \beta v_{x}, \beta v_{y}\right) \\
& =\frac{2 N e^{-\alpha \sigma^{2}}}{1-e^{-\alpha r^{2}}}\left\{2 \alpha^{2} \beta\left[A(x-\xi)^{2}+2 B(x-\xi)(y-\eta)+C(y-\eta)^{2}\right]-\alpha \beta(A+C)\right\} \\
& -F\left(x, y, \beta v+z_{0}, \beta v_{x}, \beta v_{y}\right) \\
& \geqq \frac{2 N e^{-\alpha \sigma^{2}}}{1-e^{-\alpha r^{2}}}\left\{\frac{4 \alpha^{2} \beta\left(A C-B^{2}\right) \sigma^{2}}{A+C+\left[(A+C)^{2}-4\left(A C-B^{2}\right)\right]^{1 / 2}}-\alpha \beta(A+C)\right\} \\
& -H_{N+\left|z_{0}\right|}\left(\beta^{2} v_{x}^{2}+\beta^{2} v_{y}^{2}\right)
\end{aligned}
$$

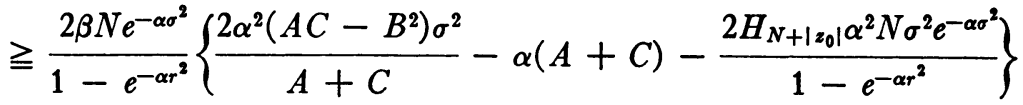

$$
\begin{aligned}
& \geqq \frac{2 \alpha \beta N e^{-\alpha \sigma^{2}}}{(A+C)\left(1-e^{-\alpha r^{2}}\right)}\left\{2 \alpha \epsilon^{2} r^{2} d-\delta^{2}-2 \delta H_{N+\left|z_{0}\right|} N M\right\}
\end{aligned}
$$

where $\delta \equiv \operatorname{Max}[a(p, q)+c(p, q)]$ for $p^{2}+q^{2} \leqq 1$ and $M \equiv \operatorname{Max} t e^{-\epsilon^{2} t} /\left(1-e^{-t}\right)$ for $0 \leqq t<+\infty$. Thus there is a fixed constant $\gamma>0$ independent of $r,(\xi, \eta)$, and $\beta$ such that

$$
L[\beta v]-F\left(x, y, \beta v+z_{0}, \beta v_{x}, \beta v_{y}\right) \geqq 0
$$

in $S(\xi, \eta, r)$ when $\alpha=\gamma / 2 \epsilon^{2} r^{2} d$.

Now assume that $R$ the radius of the disk $K$ is chosen so that

$$
3 R / 4 \geqq \frac{2 N}{\epsilon}=\frac{w(0,0)}{\epsilon} .
$$

Set 


$$
v_{1}(x, y) \equiv v\left(x, y ; x_{1}, y_{1}, 2 R\right)
$$

and

$$
v_{2}(x, y) \equiv v\left(x, y ; x_{2}, y_{2}, 2 R\right) .
$$

Recall that each point $(x, y) \in K_{1} \cap K_{2}$ satisfies at least one of the previously listed conditions (a), (b), or (c). Assume that $(x, y)$ is in the interior of $K_{1} \cap K_{2}$ and that either $(x, y) \in \Gamma$ or $(x, y)$ satisfies condition (b), then we can apply the Maximum Principle to conclude that $w(x, y) \geqq v_{1}(x, y)$. Similarly, if $(x, y)$ is in the interior of $K_{1} \cap K_{2}$ and either $(x, y) \in \Gamma$ or $(x, y)$ satisfies (c), we can conclude $w(x, y) \geqq v_{2}(x, y)$. Thus for all $(x, y) \in \operatorname{int}\left(K_{1} \cap K_{2}\right)$ it is true that

$$
w(x, y) \geqq \operatorname{Min}\left[v_{1}(x, y), v_{2}(x, y)\right] .
$$

Now on the circle $\left(x-x_{0} / 4\right)^{2}+\left(y-y_{0} / 4\right)^{2}=\epsilon^{2}(3 R / 4)^{2}$

$$
\beta_{0} \equiv \operatorname{Min}\left[v_{1}(x, y), v_{2}(x, y)\right]=\frac{N\left[e^{-\gamma \rho^{2} / 2 \epsilon^{2} d}-e^{-\gamma / 2 \epsilon^{2} d}\right]}{1-e^{-\gamma / 2 \epsilon^{2} d}}
$$

where $\rho=(61)^{1 / 2} / 8+3 \epsilon / 8<1$. Another application of the Maximum Principle yields

$$
w(x, y) \geqq \beta_{0} v_{3}(x, y) / N
$$

on $S\left(x_{0} / 4, y_{0} / 4,3 R / 4\right)$ where

$$
v_{3}(x, y) \equiv v\left(x, y ; x_{0} / 4, y_{0} / 4,3 R / 4\right) .
$$

The annulus $S\left(x_{0} / 4, y_{0} / 4,3 R / 4\right)$ contains the disk with center at $(0,0)$ and radius $(1 / 4-3 \epsilon / 4) R$. On this disk

$$
\frac{\beta_{0}}{N} v_{3}(x, y) \geqq \frac{\beta_{0}\left[e^{-\gamma \tau^{2} / 2 \epsilon^{2} d}-e^{-\gamma / 2 \epsilon^{2} d}\right]}{1-e^{-\gamma / 2 \epsilon^{2} d}}
$$

where $\tau=2 / 3-\epsilon$.

We conclude that there is a fixed $\beta_{1}, 0<\beta_{1}<1$, independent of $R$ such that

$$
w(x, y) \geqq \beta_{1} w(0,0)
$$

on the disk with center at $(0,0)$ and radius $(1 / 4-3 \epsilon / 4) R$. Since $R$ can be taken as large as we please, we conclude that

$$
w(x, y) \geqq \beta_{1} w(0,0)
$$

everywhere. However, this contradicts the fact that glb $w(x, y)=0$ and we conclude that a solution of (2) which is bounded above or below must be a constant. 


\section{REFERENCES}

1. L. V. Ahlfors, Complex analysis, McGraw-Hill, New York, 1953.

2. S. N. Bernstein, Über ein geometrische Theorem und seine Anwendung auf die partiellen Differentialgleichungen vom elliptischen Typus, Math. Z. 26 (1927), 551-558.

3. J. B. Serrin, Jr., On the Harnack inequality for linear elliptic equations, J. Analyse Math. 4 (1955-1956), 292-308.

4. L. Bers and L. Nirenberg, On linear and nonlinear elliptic boundary value problems in the plane, Convegno Internationale sulle Equazioni Derivate Parziali (Trieste, 1954), pp. 141-167, Edizioni Cremonese, Rome, 1955.

5. E. Hopf, Elementarie Betrachtungen über die Losungen partieller Differentialgleichungen zweiter Ordnung vom elliptischen Typus, Sitzungsberichte Preuss. Akad. Wiss. 19 (1927), 147152.

Bowling Green State University, Bowling Green, OHio

UNIVERSITY OF NEBRASKA,

LiNCOLN, NebRASKa 